\title{
A Microwave-Based Chemical Factory in the Lab: From Milligram to Multigram Preparations
}

\author{
Laura Rinaldi, ${ }^{1,2}$ Diego Carnaroglio, ${ }^{1}$ Laura Rotolo, ${ }^{1}$ and Giancarlo Cravotto ${ }^{1}$ \\ ${ }^{1}$ Dipartimento di Scienza e Tecnologia del Farmaco and Centre for Nanostructured Interfaces and Surfaces (NIS), University of Turin, \\ Via P. Giuria 9, 10125 Torino, Italy \\ ${ }^{2}$ Dipartimento di Biotecnologie Molecolari e Scienze per la Salute, University of Turin, Via Quarello 15, 10135 Torino, Italy
}

Correspondence should be addressed to Giancarlo Cravotto; editor.cravotto@unito.it

Received 5 January 2015; Accepted 8 February 2015

Academic Editor: Augusto C. Tome

Copyright (C) 2015 Laura Rinaldi et al. This is an open access article distributed under the Creative Commons Attribution License, which permits unrestricted use, distribution, and reproduction in any medium, provided the original work is properly cited.

\begin{abstract}
Microwave technology is changing the way we design and optimize synthetic protocols and their scaling up to multigram production levels. The latest generation of dedicated microwave reactors enables operators to quickly screen reaction conditions by means of parallel tests and select the best catalyst, solvent, and conditions. Pilot scale synthetic procedures require flow-through conditions in microwave flow reactors which can be obtained by adapting classic batch protocols. Microwave-assisted chemical processes play a pivotal role in the design of sustainable multigram preparations which address the double requirement of process intensification and competitive production costs. Although most researchers are likely to be acquainted with the great potential of dielectric heating, the advantages and disadvantages of a particular device or the conditions needed to maximize efficiency and functionality are often overlooked. The double aims of the present review are to provide a panoramic snapshot of commercially available lab microwave reactors and their features as well as highlighting a few selected applications of microwave chemistry of particular relevance.
\end{abstract}

\section{Introduction}

Microwave (MW) technology is a well-established technique in organic, inorganic, medicinal, analytical, and materials chemistry. Practitioners are referred to a series of general reviews and monographs for in-depth coverage of these subjects [1-4].

Since the mid-1970s, professional MW reactors found in analytical laboratories have mainly been used for sample preparation (i.e., digestion, extraction, dissolution, etc.) [5]. $\mathrm{MW}$-assisted organic synthesis (MAOS) and MW-assisted extraction (MAE) have also become very popular over the last decade. MAOS, together with a few other enabling technologies, has dramatically changed the way we consider synthetic processes [1]. Since the first application of MW irradiation to promote chemical reactions in $1986[6,7]$, the number of scientific papers and books in this exciting field has sharply increased [8].
The term "enabling techniques" includes all nonconventional energy sources that may enhance chemical conversions [9]. Besides dielectric heating [10], ultrasound (US), hydrodynamic cavitation (HC), ball-milling, and microfluidic systems also demonstrate huge process intensification potential $[11,12]$. The impressive advantages of MW technology have been reported in drug discovery [13], materials science, polymer chemistry [14], nanotechnology [15], organic synthesis, and bioconjugation fields [16]. Ever since van Noorden's 2008 report entitled "Microwaving myths" [17], in which several experts described the current status of MW-assisted chemistry, was published, new technological advances have been made that have broadened the applications of these techniques.

The market offers two types of MW instrumentations classified as monomode and multimode reactors. The latter have a long history and are the direct evolution of the domestic ovens introduced to the US market in 1967. 
Multimode ovens have a large cavity and relatively low field density, while monomode reactors have a small cavity which is directly installed in the waveguide, allowing for high power input. Monomode devices have been mainly used to study reaction pathways on a mmol-mg scale [18]. R\&D departments often need to synthesize compounds on gram/multigram scale for catalytic, toxicological, and biological tests. This can be carried out in multimode reactors. The diffusion of pressurized MW reactors has extended the range of operating temperature, enabling the use of low-boiling organic solvents and reagents. In stubborn reactions, the use of sealed vessels replaced high boiling solvents (xylene, $\mathrm{N}$ methyl-2-pyrrolidone, etc.) with common organic solvents. High pressures and temperatures require efficient cooling, if side reactions are to be avoided and automatic fail-safe emergency functions are needed as well. Several pressurized vessels (till 40 bar) are in the market as dedicated rotors to perform parallel synthesis. A more recent achievement in MW instrumentation is the single reaction chamber (SRC, $1.5 \mathrm{~kW} / 1.5 \mathrm{~L}$ ), an innovative device well suited for $\mathrm{mg}$ to multigram scale reactions $[19,20]$.

\section{Professional Devices}

The diffusion of professional equipment for MAOS began in the ' 90 s, thus paving the way for new promising applications. A French company, Prolabo, produced the first professional MW device [21] and a few years later CEM Corporation (USA) acquired all Prolabo microwave-based product lines. Over the last two decades, several dedicated innovative reactors have been developed by Milestone (Italy), MLS GmbH (Germany), Anton Paar (Austria), CEM, Biotage (Sweden), and Sairem (France). More recently a few other companies, mainly from Asian countries, have joined the market.

2.1. Multimode Ovens. Multimode ovens were the first professional devices produced for analytical and organic synthesis laboratories. They have a large cavity $(\sim 40 \mathrm{~L})$ and systems that ensure the control of temperature, pressure, time, sample stirring, and postreaction cooling. A useful achievement in this line of products was the development of rotors that bear pressure-resistant vessels for simultaneous multisample irradiation, an important tool which is used both in sample digestion and organic synthesis. Some ovens mount a double magnetron (Figure 1) unit equipped with a pyramidal-shaped rotating diffuser (multimode MicroSynth, Milestone/MLS) [22].

2.2. Monomode Reactors. Over the years, technological investment has transformed the concept of "MW oven" into "MW reactor" that can boast of improved energy transfer. Monomode reactors have small compact cavities with high field MW density directly focused on the reaction mixture, resulting in exceedingly fast heating rates. Monomode reactors are well suited for fundamental studies of reaction mechanisms.

CEM, Anton Paar, and Biotage are the main companies which manufacture monomode reactors equipped with

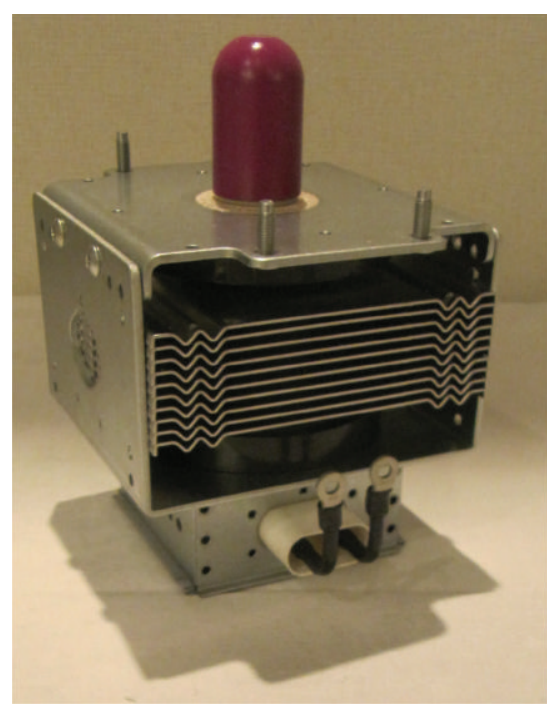

FIGURE 1: Typical water jacket—air cooled $2.45 \mathrm{GHz}$ magnetron (1.5 or $2.0 \mathrm{~kW}$, Panasonic).

autosamplers and IR or optic fibre thermometers and even a camera so operators can visually follow the reaction process [23] (Figure 2).

2.3. Dedicated Reactors. Multimode ovens have been reinvented over the years with the aim of creating a series of devices for specific applications. Milestone/MLS have developed a line of versatile instruments which exploit the technology of the classic multimode oven. RotoSynth is an innovative MW reactor with a $45^{\circ}$ tilted rotating reaction vessel. It is an excellent platform for solid phase MW synthesis as it ensures good solid mixing and high reproducibility from $\mathrm{mg}$ to $2 \mathrm{~kg}$ of material. A vacuum can be applied during the process allowing solvents or volatile products to evaporate.

FlowSynth is a laboratory scale MW reactor which can translate batch processes into semibatch or continuous flow processes. The reaction chamber consists of a PTFE tube of variable capacity protected by a quartz-fibre reinforced PEEK sheath which is vertically mounted in the MW cavity. The reaction mixture is pumped by a high-pressure membrane pump from the base upwards through the column to the top regulated by backpressure valve. The FlowSynth can operate at temperatures of up to $230^{\circ} \mathrm{C}$ and pressures of up to $30 \mathrm{bar}$ with flow rates between 10 and $200 \mathrm{~mL} / \mathrm{min}$. It can process significant quantities of material and furnishes many $\mathrm{kg}$ of product per day.

2.3.1. Kilogram Scale Reactors. Laboratory productivity has increased thanks to dedicated MW devices, mainly from Milestone/MLS, Anton Paar, and Biotage. These devices give $\mathrm{kg}$ per day scale productivity in a batch configuration. Safe and easy procedures are very good answers to the problem of scaling up chemical processes. They are specifically designed so that methods developed in lab-scale systems can be safely and predictably transferred to larger scales without reoptimization. In addition to homogeneous reactions, 


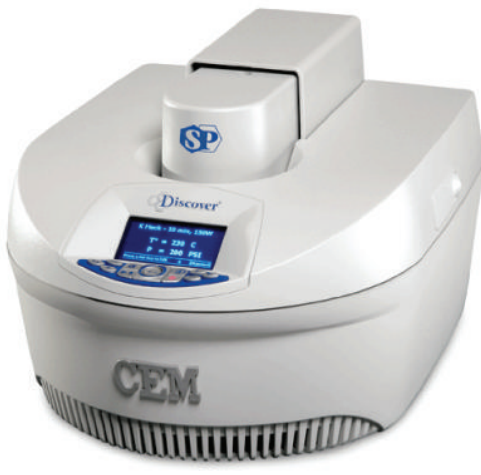

(a)

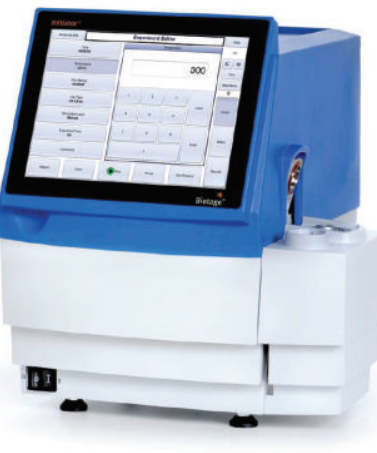

(b)

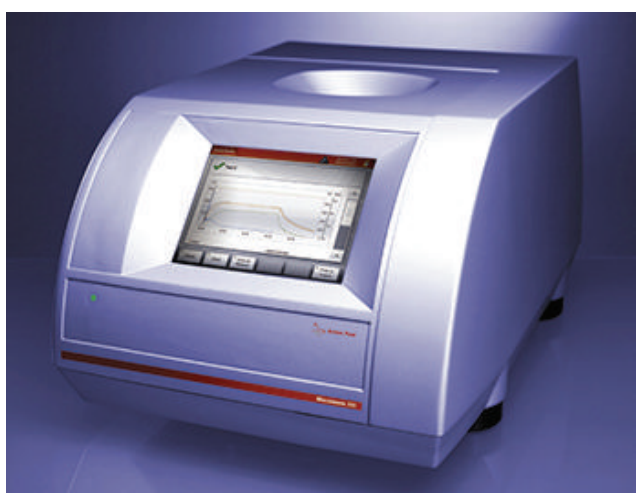

(c)

Figure 2: Discover (CEM); Initiator (Biotage); and Monowave 300 (Anton Paar).

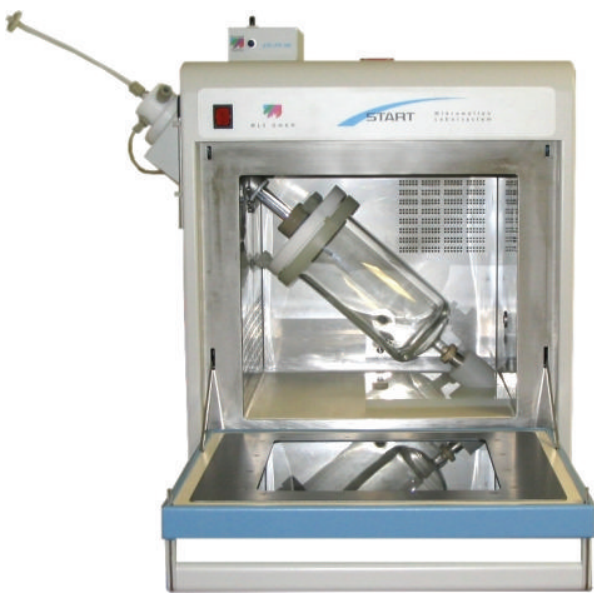

(a)

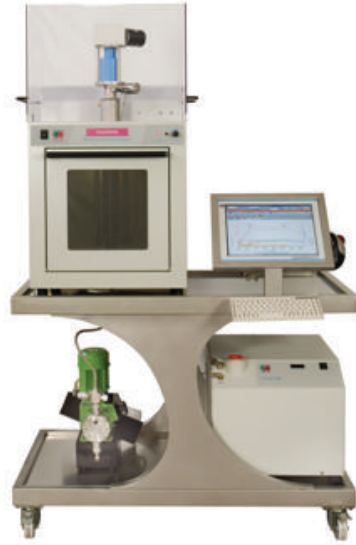

(b)

Figure 3: RotoSynth and FlowSynth (Milestone/MLS).

the system also supports heterogeneous reactions and the use of solid catalysts, heavy slurries, and crystallizing products. An important feature that has been added by Milestone/MLS to the UltraClave (Figure 4(a)) is MW irradiation with highpressure vessel technology, enabling researchers to conduct batch reactions at 200 bar. Moreover, UltraClave is the biggest lab-scale MW reactor commercially available. The $3.5 \mathrm{~L}$ reaction chamber enables several samples (it is designed to hold 6 to 77 individual sample containers made of glass, quartz, or TFM) or a single large reaction mixture to be processed. The Masterwave BTR reactor (Anton Paar) (Figure 4(c)) and the Advancer (Biotage) (Figure 4(b)) are designed to perform reactions in a sealed vessel with a maximum working volume of $1 \mathrm{~L}$ and $300 \mathrm{~mL}$, respectively.

\subsubsection{Equipment for $M W$-Assisted Extraction (MAE) and} $M W$-Assisted Hydrodistillation (MAH). The principles of MAE are different from those of conventional methods, such as solid-liquid or simple extraction. In fact, the electromagnetic waves cause changes in cell structure and consequently the extraction process: heat and mass gradients work in the same direction [2]. In conventional extractions, mass transfer occurs from the inside to the outside, although the heat transfer occurs in the opposite direction. Over the last decade, MAE [24] and MAH [25] have proven to be the most promising techniques for improving plant extraction and hydrodistillation. The first MW technique for the extraction of essential oils from aromatic plants and spices was introduced by Craveiro et al. [26] in 1989. Milestone Technologies developed a series of new MW-dedicated instruments for MAE and MAH: Neos, Neos-GR, and MAC-75 (Figure 5). Neos-GR uses techniques patented by Chemat et al. for hydrodiffusion and gravity extraction [27]. This technique consists of placing a matrix in a reactor inside the MW oven without adding water or organic solvents. Essential oils as well as the internal water of the matrix are recovered from the bottom of the reactor via condensation of the distillate. MAC75 is a dedicated pilot unit from Milestone Technologies for the industrial production of essential oils or extracts on small scales (75 L rotating drum).

2.4. Latest Generation Reactors. The SRC (single reaction chamber) is one of the most recent innovations in $\mathrm{MW}$ 


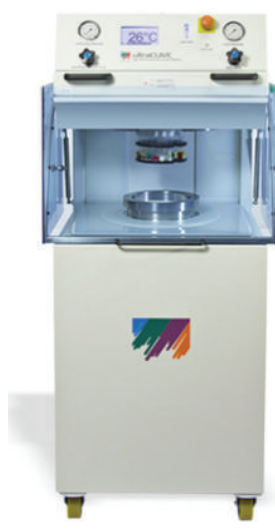

(a)

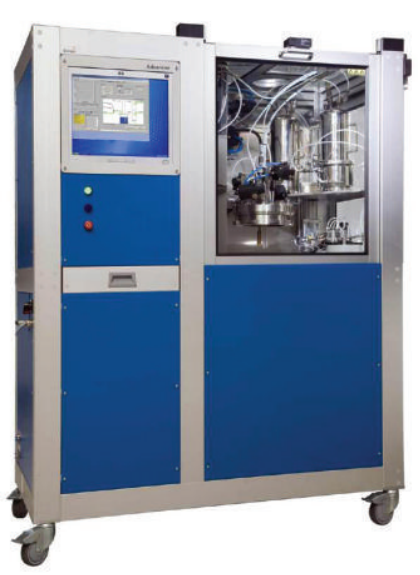

(b)

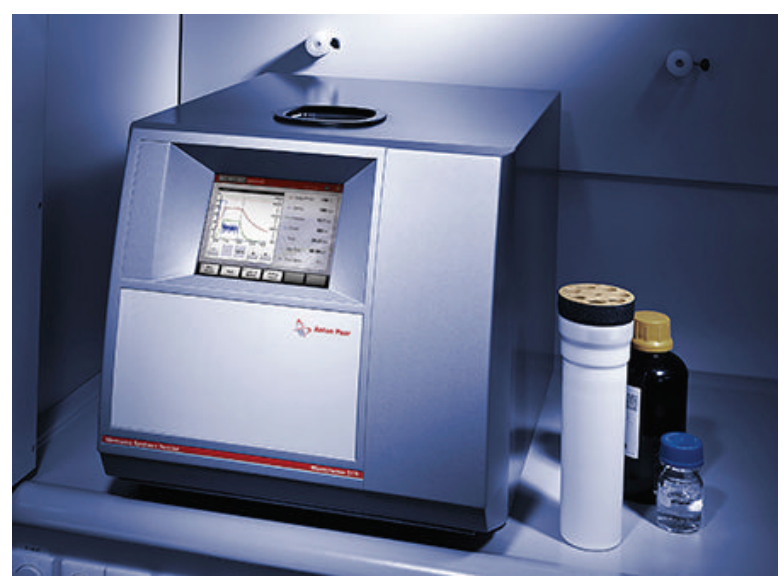

(c)

FIGURE 4: UltraClave (Milestone/MLS), Advancer (Biotage), and Masterwave BTR (Anton Paar).

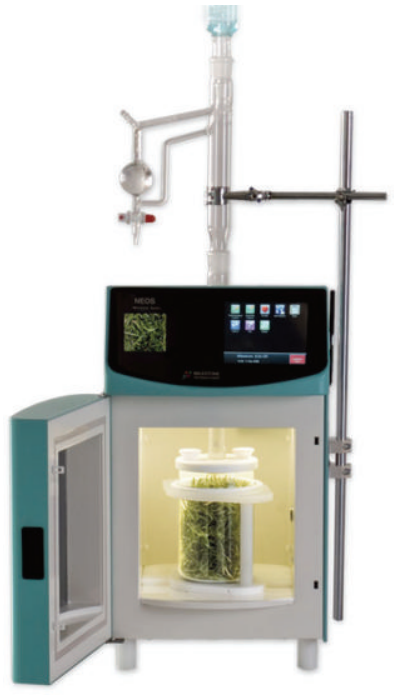

(a)

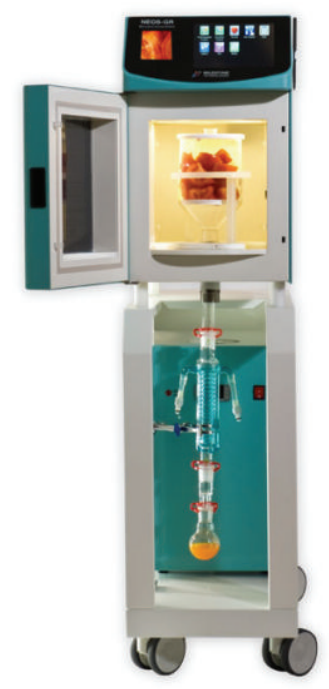

(b)

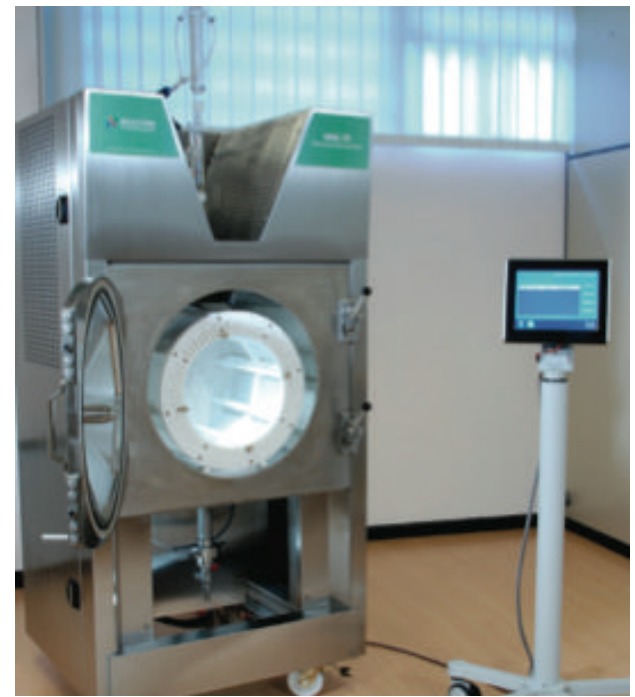

(c)

Figure 5: Neos, Neos-GR, and MAC-75 (Milestone Technologies).

instrumentation to move from sealed vessels to sealed cavities. SRC allows catalysts and reaction conditions to be easily screened as several tubes can be irradiated simultaneously on a single rack at the same pressure and temperature even with different solvents (Figure 6). This facilitates the search for optimal catalyst load, solvent ratio, and type. Besides these features, the SynthWave reactor (Milestone/MLS) is equipped with multiple gas inlets and is designed to work over a wide range of temperatures and pressures (up to $300^{\circ} \mathrm{C}$ and $199 \mathrm{bar}$, power density $1.5 \mathrm{~kW} / \mathrm{L}$ ). The efficient magnetic/mechanic stirring systems, from a few $\mathrm{mL}$ up to the $1 \mathrm{~L}$ vessel, are well suited to heterogeneous reacting mixtures, including a wide range of low-boiling-point reagents and solvents. Extremely efficient reaction vessel cooling avoids or minimizes product degradation and side reactions.

\section{Applications}

The huge number of MW applications in organic chemistry shows the growing interest that synthetic chemists confer to this technology $[9,28,29]$. A number of innovative dedicated reactors designed for specific applications enable all processing conditions and parameters to be strictly controlled [30]. Three different applications of high scientific relevance and economical impact have been selected for this section. These $\mathrm{MW}$-assisted synthetic processes are the $\mathrm{C}-\mathrm{C}$ cross-coupling reaction, biodiesel production and biomass conversion, and the functionalization of nanomaterials.

3.1. C-C Cross-Coupling Reactions. Carbon-carbon coupling reactions are certainly one of the key reactions in organic 


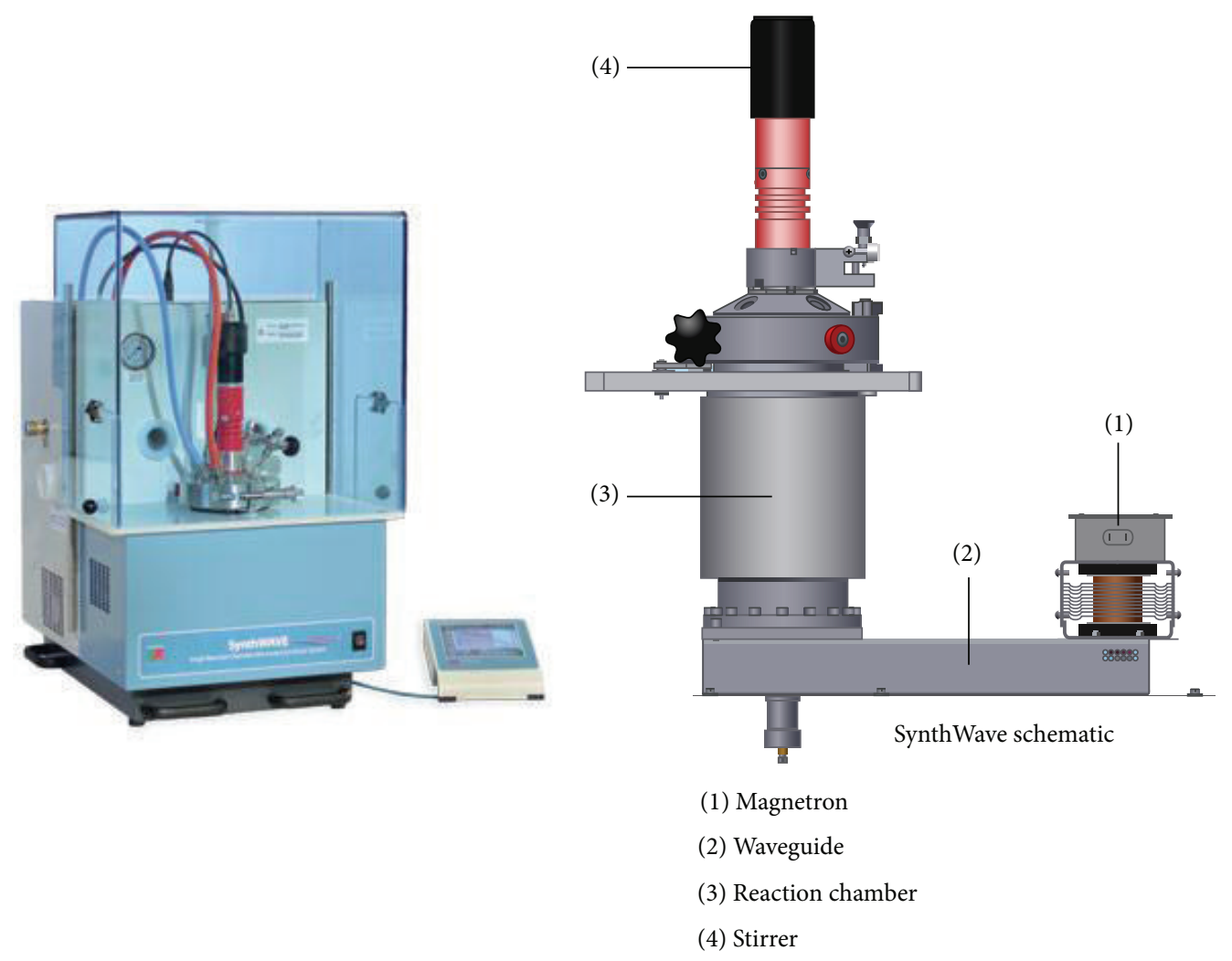

(a)

(b)

FIgURE 6: SynthWave (Milestone/MLS).

synthesis [31]. Heck [32] and Suzuki [33] reactions, widely used for $\mathrm{C}-\mathrm{C}$ bond formation, are generally catalyzed by soluble palladium complexes in the presence of ligands. In 1996, Larhed and Hallberg [34] proposed the first example of cross-coupling reactions enhanced by MW irradiation using a monomode reactor. Later, Leadbeater and Marco [35] developed a methodology for ligand-free MW-mediated Suzuki-Miyaura coupling in aqueous media using a CEM Discover Unit. The authors scaled up the reactions from $10 \mathrm{~mL}$ sealed tubes to larger open vessels in a multimode oven equipped with double-surface reflux condenser [36, 37]. Owing to the poor penetration depth of MW, only few centimetres in most solvents, Moseley and coworker introduced a continuous flow system [38] for the Heck reaction in a FlowSynth (Milestone/MLS) (Figure 3(b)). Over the last decade, huge efforts have been put into the search for cost-effective, ligand-free Pd catalysts and new activation techniques for highly efficient C-C couplings [39]. Simple Pd salts, metals supported on active charcoal, or metal-loaded polymers have all been efficiently used under MW irradiation $[40,41]$ and even under simultaneous irradiation with US which tends to give better results as shown for Heck reactions with very low ligandless catalyst loads [42]. Suzuki crosscoupling reactions have also been efficiently performed in glycerol under simultaneous MW/US irradiation [43]. More recently, the new MW reactor, SynthWave, allowed for fast catalysts/solvent screening by means of parallel reactions.
A novel solid cross-linked cyclodextrin based catalyst obtained via reticulation with hexamethylene diisocyanate in solutions containing Pd(II) cations was used in Heck and Suzuki reactions [44]. This system, equipped with a rack of several test tubes, facilitated the search for optimal catalyst load, solvent ratio, and type and ensured high energy density transfer.

3.2. Biodiesel Production and Biomass Conversion. Over the last 30-40 years, several renewable energy sources have been developed. Biodiesel plays an important role here because of its high heating values, which are nearly equivalent to diesel fuels, and its low overall environmental impact. The sustainability of the process is strongly related to the type of oil source and overall energy consumption [45]. MW has been proven to dramatically enhance the transesterification of triglycerides because it improves heat transfer as compared to conventional methods [46]. The first MW-assisted protocol for oil transesterification was reported in 2006 [47]. The reaction was carried out at atmospheric pressure in few minutes using a lower oil/methanol (or ethanol) ratio than typical methods under conventional heating. Experiments were carried out for a few millilitres both in a monomode reactor and on a large scale of up to $3 \mathrm{~kg}$ of oil using multimode apparatus. Continuous flow preparations have also been studied for industrial applications with energy saving being the main goal $[48,49]$. The combined use of 
MW and US [50] has also been investigated in flow pilots and industrial plants [51]. MW not only is useful as an alternative heating method, but also produces biochar and bio-oil at relatively low temperatures $\left(<200^{\circ} \mathrm{C}\right)$ via different pyrolytic mechanisms $[52,53]$. Besides the use of traditional MW ovens [54-56], a big step forward has come from the combination of MW/US [57, 58] and new dedicated reactors. Biomass is recognized as a major renewable source that can be used to produce biofuels and biochemicals. The peculiar properties of MW used as a source of volumetric and selective dielectric heating can be efficiently exploited for the hydrolysis of biopolymers and their further conversion to highly valuable fine chemicals. Budarin et al. [59] highlighted the high potential of MW in the treatment of biomass. In their work, the use of RotoSynth (Milestone/MLS) (Figure 3(a)) was particularly profitable in process scale up. This reactor, equipped with rotating vessel under vacuum, ensures that MW energy is uniformly distributed while offering liquid product fractionation capabilities. Tabasso et al. [16] have reported a MW protocol for the flash conversion of nonedible polysaccharides and postharvest tomato plant waste to levulinic acid using a SRC system $\left(225^{\circ} \mathrm{C}\right.$ for only $\left.2 \mathrm{~min}\right)$. High levulinic acid yields were obtained under the same conditions from nonedible polysaccharides such as cellulose and chitosan (90\% and 95\%, resp.). When performed at lower temperatures $\left(\leq 150^{\circ} \mathrm{C}\right)$, the same process favoured the selective hydrolysis of carbohydrates to simple monosaccharides, thus demonstrating the versatility of the procedure which can selectively give either simple sugars or pure levulinic acid simply by varying the reaction temperature.

3.3. Functionalization of Nanomaterials. Nanoparticles have a large surface to volume ratio, consequently display increased surface activity over bulk materials, and fill potential applications in many fields, especially in catalysis [60]. The properties of nanoparticles widely depend on their size and shape; the introduction of MW to their synthesis has allowed small uniform nanoparticles to be obtained [61]. Constant conditions, which are easily addressed under MW, are crucial during nucleation and nanomaterial growth and augment the reaction rate. All MW system types have been used for the synthesis of nanoparticles, including domestic ovens and several dedicated reactors [3]. Carbon nanotubes (CNTs) have become key protagonists over the last two decades $[62,63]$ because of their unique physical and chemical properties [64]. Under conventional heating CNTs show poor reactivity and require prolonged heating under reflux, occasionally for days, to achieve an acceptable functionalization degree. CNTs strongly absorb MW radiation and enable faster raw material purification and sidewall chemical grafting even in large-scale protocols $[65,66]$. Negra et al. [67] have described the preparation of PEG grafted singlewall carbon nanotubes (SWCNs) using a multimode reactor (ETHOS 1600), which promoted the reaction more intensely than conventional heating. Of the most promising procedures for SWCNs functionalization, MW-assisted cycloadditions' high efficiency has set them apart. Nice examples can be found in fullerene grafting with a pyridyl group [68] and the 1,3-dipolar cycloaddition reaction of a nitrile oxide with SWNTs [69]. The SWNTs obtained were in the range of hundreds of nanometers, kept their tube morphology, and showed a trend for reduced aggregation, all in only $45 \mathrm{~min}$. A simple and efficient protocol for the preparation of highly functionalized SWCTs makes use of the SynthWave reactor [70]. It is a solvent-free reaction which generates carbonyl ylides from a series of oxiranes. The SRC instrument performs the reaction at under $20 \mathrm{bar}$ of $\mathrm{N}_{2}$, avoids air oxidation, and gives a homogenous and regular solid sample heating because the reaction vessel is immersed in water, which acts as an adsorbing medium, and the stirring is very efficient. Another example is the efficient grafting of silica particles with $\beta$-cyclodextrins [71]. The study demonstrated that MW irradiation can promote all the steps in the synthetic route allowing for loading values that are three times higher than silica derivatized in an oil bath $(135 \mu \mathrm{mol} / \mathrm{g}$ versus $47 \mu \mathrm{mol} / \mathrm{g})$. The high efficiency of these grafting procedures will pave the way for novel applications.

\section{Conclusion}

The use of MW irradiation has become increasingly popular in almost all fields of chemistry research and in particular in MAOS. As reported by J. Moseley (Astra Zeneca), "virtually all new compounds now have their first synthesis in a microwave." In fact, MW has doubtlessly given the most impressive contribution of all the many enabling technologies for synthetic process activation and intensification that are available. The most recent achievements take advantage of the latest generation of professional reactors which have been especially designed for extreme reaction conditions under strict monitoring control. High versatility means that there are no limits on the composition of the reaction mixture, which can include solids and gases, and can provide milligram to multigram scale synthetic processes in suitable batch and flow reactors.

\section{Conflict of Interests}

The authors declare that there is no conflict of interests regarding the publication of this paper.

\section{Acknowledgment}

The University of Turin is acknowledged for its financial support (Fondi Ricerca Locale 2013).

\section{References}

[1] A. De La Hoz and A. Loupy, Microwaves in Organic Synthesis, Springer Science, New York, NY, USA, 3rd edition, 2012.

[2] F. Chemat and G. Cravotto, Microwave-Assisted Extraction for Bioactive Compounds: Theory and Practice, vol. 4 of Food Engineering Series, Springer, New York, NY, USA, 2013.

[3] S. Horikoshi and N. Serpone, Nanoparticle Synthesis-Fundamentals and Applications, Wiley-VCH, Weinheim, Germany, 2013. 
[4] Z. Fang, R. L. Smith, and X. Qi, Production of Biofuels and Chemicals with Microwave, vol. 3 of Biofuels and Biorefineries, Springer Science, New York, NY, USA, 2014.

[5] L. Chen, D. Song, Y. Tian, L. Ding, A. Yu, and H. Zhang, "Application of on-line microwave sample-preparation techniques," Trends in Analytical Chemistry, vol. 27, no. 2, pp. 151-159, 2008.

[6] R. Gedye, F. Smith, K. Westaway et al., "The use of microwave ovens for rapid organic synthesis," Tetrahedron Letters, vol. 27, no. 3, pp. 279-282, 1986.

[7] R. J. Giguere, T. L. Bray, S. M. Duncan, and G. Majetich, "Application of commercial microwave ovens to organic synthesis," Tetrahedron Letters, vol. 27, no. 41, pp. 4945-4948, 1986.

[8] C. O. Kappe and D. Dallinger, "Controlled microwave heating in modern organic synthesis: highlights from the 2004-2008 literature," Molecular Diversity, vol. 13, no. 2, pp. 71-193, 2009.

[9] R. B. N. Baig and R. S. Varma, "Alternative energy input: mechanochemical, microwave and ultrasound-assisted organic synthesis," Chemical Society Reviews, vol. 41, no. 4, pp. 15591584, 2012.

[10] C. O. Kappe, "Controlled microwave heating in modern organic synthesis," Angewandte Chemie International Edition, vol. 43, no. 46, pp. 6250-6284, 2004.

[11] G. Cravotto and P. Cintas, "The combined use of microwaves and ultrasound: improved tools in process chemistry and organic synthesis," Chemistry, vol. 13, no. 7, pp. 1902-1909, 2007.

[12] G. Cravotto and P. Cintas, "The combined use of microwaves and ultrasound: methods and practice," in Microwaves in Organic Synthesis, A. De La Hoz and A. Loupy, Eds., vol. 1, Springer, New York, NY, USA, 3rd edition, 2012.

[13] D. Garella, E. Borretto, A. Di Stilo, K. Martina, G. Cravotto, and P. Cintas, "Microwave-assisted synthesis of $N$-heterocycles in medicinal chemistry," MedChem Comm, vol. 4, no. 10, pp. 13231343, 2013.

[14] K. Kempe, C. R. Becer, and U. S. Schubert, "Microwaveassisted polymerizations: recent status and future perspectives," Macromolecules, vol. 44, no. 15, pp. 5825-5842, 2011.

[15] R. S. Varma, "Greener routes to organics and nanomaterials: sustainable applications of nanocatalysts," Pure and Applied Chemistry, vol. 85, no. 8, pp. 1703-1710, 2013.

[16] S. Tabasso, E. Montoneri, D. Carnaroglio, M. Caporaso, and G. Cravotto, "Microwave-assisted flash conversion of nonedible polysaccharides and post-harvest tomato plant waste to levulinic acid," Green Chemistry, vol. 16, no. 1, pp. 73-76, 2014.

[17] R. van Noorden, "Microwaving myths," Chemistry World, vol. 5, pp. 40-46, 2008.

[18] C. O. Kappe and A. Stadler, Microwaves in Organic and Medicinal Chemistry, Wiley-VCH, Weinheim, Germany, 2005.

[19] P. Cintas, D. Carnaroglio, L. Rinaldi, and G. Cravotto, "Complementary and synergic effects of microwaves and ultrasound in metal-assisted synthesis," Chemistry Today, vol. 30, no. 3, pp. 33-35, 2012.

[20] V. Hessel, G. Cravotto, P. Fitzpatrick, B. S. Patil, J. Lang, and W. Bonrath, "Industrial applications of plasma, microwave and ultrasound techniques: nitrogen-fixation and hydrogenation reactions," Chemical Engineering and Processing, vol. 71, pp. 1930, 2013.

[21] A. Loupy, P. Pigeon, and M. Ramdani, "Synthesis of long chain aromatic esters in a solvent-free procedure under microwaves," Tetrahedron, vol. 52, no. 19, pp. 6705-6712, 1996.

[22] D. Rossi, M. Urbano, A. C. Baraglia et al., "Polymer-assisted solution-phase synthesis under combined ultrasound and microwave irradiation: preparation of $\alpha, \beta$-unsaturated esters and carboxylic acids, key intermediates of novel sigma ligands," Synthetic Communications, vol. 39, no. 18, pp. 3254-3262, 2009.

[23] M. A. Herrero, J. M. Kremsner, and C. O. Kappe, "Nonthermal microwave effects revisited: on the importance of internal temperature monitoring and agitation in microwave chemistry," Journal of Organic Chemistry, vol. 73, no. 1, pp. 36-47, 2008.

[24] L. Alexandru, L. Pizzale, L. Conte, A. Barge, and G. Cravotto, "Microwave-assisted extraction of edible Cicerbita alpina shoots and its LC-MS phenolic profile," Journal of the Science of Food and Agriculture, vol. 93, no. 11, pp. 2676-2682, 2013.

[25] L. Orio, G. Cravotto, A. Binello, G. Pignata, S. Nicola, and F. Chemat, "Hydrodistillation and in situ microwave-generated hydrodistillation of fresh and dried mint leaves: a comparison study," Journal of the Science of Food and Agriculture, vol. 92, no. 15, pp. 3085-3090, 2012.

[26] A. A. Craveiro, F. J. A. Matos, J. W. Alencar, and M. M. Plumel, "Microwave oven extraction of an essential oil," Flavour and Fragrance Journal, vol. 4, no. 1, pp. 43-44, 1989.

[27] F. Chemat, M. Vian, and F. Visinoni, "Microwave Hydrodiffusion for Isolation of Natural Products," US 20100062121 A1, 2008.

[28] A. Sharma, D. Vacchani, and E. van der Eycken, "Developments in direct $\mathrm{C}-\mathrm{H}$ arylation of (hetero)arenes under microwave irradiation," Chemistry, vol. 19, no. 4, pp. 1158-1168, 2013.

[29] C. O. Kappe, "Reply to the correspondence on microwave effects in organic synthesis," Angewandte Chemie-International Edition, vol. 52, no. 31, pp. 7924-7928, 2013.

[30] C. O. Kappe, B. Pieber, and D. Dallinger, "Microwave effects in organic synthesis: myth or reality?" Angewandte ChemieInternational Edition, vol. 52, no. 4, pp. 1088-1094, 2013.

[31] K. C. Nicolaou and E. J. Sorensen, Classics in Total Synthesis, Wiley-VCH, Weinheim, Germany, 1996.

[32] R. F. Heck and J. P. Nolley Jr., "Palladium-catalyzed vinylic hydrogen substitution reactions with aryl, benzyl, and styryl halides," Journal of Organic Chemistry, vol. 37, no. 14, pp. 2320$2322,1972$.

[33] N. Miyaura and A. Suzuki, "Palladium-catalyzed cross-coupling reactions of organoboron compounds," Chemical Reviews, vol. 95, no. 7, pp. 2457-2483, 1995.

[34] M. Larhed and A. Hallberg, "Microwave-promoted palladiumcatalyzed coupling reactions," Journal of Organic Chemistry, vol. 61, no. 26, pp. 9582-9584, 1996.

[35] N. E. Leadbeater and M. Marco, "Ligand-free palladium catalysis of the suzuki reaction in water using microwave heating," Organic Letters, vol. 4, no. 17, pp. 2973-2976, 2002.

[36] N. E. Leadbeater and M. Marco, "Transition-metal-free Suzukitype coupling reactions: scope and limitations of the methodology," Journal of Organic Chemistry, vol. 68, no. 14, pp. 56605667, 2003.

[37] N. E. Leadbeater, V. A. Williams, T. M. Barnard, and M. J. Collins Jr., "Open-vessel microwave-promoted Suzuki reactions using low levels of palladium catalyst: optimization and scaleup," Organic Process Research and Development, vol. 10, no. 4, pp. 833-837, 2006.

[38] F. Bergamelli, M. Iannelli, J. A. Marafie, and J. D. Moseley, "A commercial continuous flow microwave reactor evaluated for scale-up," Organic Process Research and Development, vol. 14, no. 4, pp. 926-930, 2010.

[39] A. Barge, S. Tagliapietra, L. Tei, P. Cintas, and G. Cravotto, "Pdcatalyzed reactions promoted by ultrasound and/or microwave 
irradiation," Current Organic Chemistry, vol. 12, no. 18, pp. 15881612, 2008.

[40] S. E. S. Leonhardt, A. Stolle, B. Ondruschka et al., "Chitosan as a support for heterogeneous Pd catalysts in liquid phase catalysis," Applied Catalysis A: General, vol. 379, no. 1-2, pp. 30-37, 2010.

[41] K. Martina, S. E. S. Leonhardt, B. Ondruschka, M. Curini, A. Binello, and G. Cravotto, "In situ cross-linked chitosan $\mathrm{Cu}(\mathrm{I})$ or Pd(II) complexes as a versatile, eco-friendly recyclable solid catalyst," Journal of Molecular Catalysis A: Chemical, vol. 334, no. 1-2, pp. 60-64, 2011.

[42] G. Palmisano, W. Bonrath, L. Boffa, D. Garella, A. Barge, and G. Cravotto, "Heck reactions with very low ligandless catalyst loads accelerated by microwaves or simultaneous microwaves/ultrasound irradiation," Advanced Synthesis and Catalysis, vol. 349, no. 14-15, pp. 2338-2344, 2007.

[43] G. Cravotto, L. Orio, E. C. Gaudino, K. Martina, D. Tavor, and A. Wolfson, "Efficient synthetic protocols in glycerol under heterogeneous catalysis," ChemSusChem, vol. 4, no. 8, pp. 11301134, 2011.

[44] G. Cravotto, E. C. Gaudino, S. Tagliapietra, D. Carnaroglio, and A. Procopio, "A green approach to heterogeneous catalysis using ligand-free, metal-loaded cross-linked cyclodextrins," Green Processing and Synthesis, vol. 1, no. 3, pp. 269-273, 2012.

[45] A. Demirbaş, "Biodegradability of biodiesel and petrodiesel fuels," Energy Sources, Part A: Recovery, Utilization and Environmental Effects, vol. 31, no. 2, pp. 169-174, 2009.

[46] F. Motasemi and F. N. Ani, "A review on microwave-assisted production of biodiesel," Renewable and Sustainable Energy Reviews, vol. 16, no. 7, pp. 4719-4733, 2012.

[47] N. E. Leadbeater and L. M. Stencel, "Fast, easy preparation of biodiesel using microwave heating," Energy and Fuels, vol. 20, no. 5, pp. 2281-2283, 2006.

[48] T. M. Barnard, N. E. Leadbeater, M. B. Boucher, L. M. Stencel, and B. A. Wilhite, "Continuous-flow preparation of biodiesel using microwave heating," Energy and Fuels, vol. 21, no. 3, pp. 1777-1781, 2007.

[49] Y. Groisman and A. Gedanken, "Continuous flow, circulating microwave system and its application in nanoparticle fabrication and biodiesel synthesis," Journal of Physical Chemistry C, vol. 112, no. 24, pp. 8802-8808, 2008.

[50] M.-C. Hsiao, C.-C. Lin, Y.-H. Chang, and L.-C. Chen, "Ultrasonic mixing and closed microwave irradiation-assisted transesterification of soybean oil," Fuel, vol. 89, no. 12, pp. 3618-3622, 2010.

[51] R. Morschhäuser, M. Krull, C. Kayser et al., "Microwaveassisted continuous flow synthesis on industrial scale," Green Processing and Synthesis, vol. 1, no. 3, pp. 281-290, 2012.

[52] V. L. Budarin, Y. Zhao, M. J. Gronnow et al., "Microwavemediated pyrolysis of macro-algae," Green Chemistry, vol. 13, no. 9, pp. 2330-2333, 2011.

[53] V. L. Budarin, J. H. Clark, B. A. Lanigan, P. Shuttleworth, and D. J. Macquarrie, "Microwave assisted decomposition of cellulose: a new thermochemical route for biomass exploitation," Bioresource Technology, vol. 101, no. 10, pp. 3776-3779, 2010.

[54] R. Carrasquillo-Flores, M. Käldström, F. Schüth, J. A. Dumesic, and R. Rinaldi, "Mechanocatalytic depolymerization of dry (Ligno)cellulose as an entry process for high-yield production of furfurals," ACS Catalysis, vol. 3, no. 5, pp. 993-997, 2013.

[55] Y.-B. Huang and Y. Fu, "Hydrolysis of cellulose to glucose by solid acid catalysts," Green Chemistry, vol. 15, no. 5, pp. 10951111, 2013.
[56] X. Zhao, M. Wang, H. Liu, L. Li, C. Ma, and Z. Song, "A microwave reactor for characterization of pyrolyzed biomass," Bioresource Technology, vol. 104, pp. 673-678, 2012.

[57] K. Zhou, H. Qian, H. Zhou, and K. Zhu, "Study on technology of ultrasound-microwave assisted generats levulinic acid by hydrolysis of cornstarch," Science and Technology of Food Industry, vol. 29, no. 2, pp. 243-246, 2008.

[58] A. Hernoux-Villière, U. Lassi, T. Hu et al., "Simultaneous microwave/ultrasound-assisted hydrolysis of starch-based industrial waste into reducing sugars," ACS Sustainable Chemistry and Engineering, vol. 1, no. 8, pp. 995-1002, 2013.

[59] V. L. Budarin, P. S. Shuttleworth, M. de bruyn et al., "The potential of microwave technology for the recovery, synthesis and manufacturing of chemicals from bio-wastes," Catalysis Today, vol. 239, pp. 80-89, 2014.

[60] V. Polshettiwar and R. S. Varma, "Green chemistry by nanocatalysis," Green Chemistry, vol. 12, no. 5, pp. 743-754, 2010.

[61] M. N. Nadagouda, T. F. Speth, and R. S. Varma, "Microwaveassisted green synthesis of silver nanostructures," Accounts of Chemical Research, vol. 44, no. 7, pp. 469-478, 2011.

[62] P. M. Ajayan, "Nanotubes from Carbon," Chemical Reviews, vol. 99, no. 7, pp. 1787-1799, 1999.

[63] R. H. Baughman, A. A. Zakhidov, and W. A. De Heer, "Carbon nanotubes-the route toward applications," Science, vol. 297, no. 5582, pp. 787-792, 2002.

[64] J.-M. Bonard, J.-P. Salvetat, T. Stöckli, W. A. de Heer, L. Forró, and A. Chatelain, "Field emission from single-wall carbon nanotube films," Applied Physics Letters, vol. 73, no. 7, pp. 918920, 1998.

[65] F. Langa and P. De La Cruz, "Microwave irradiation: an important tool to functionalize fullerenes and carbon nanotubes," Combinatorial Chemistry and High Throughput Screening, vol. 10, no. 9, pp. 766-782, 2007.

[66] E. Vázquez and M. Prato, "Carbon nanotubes and microwaves: interactions, responses, and applications," ACS Nano, vol. 3, no. 12, pp. 3819-3824, 2009.

[67] F. D. Negra, M. Meneghetti, and E. Menna, "Microwave-assisted synthesis of a soluble single wall carbon nanotube derivative," Fullerenes, Nanotubes and Carbon Nanostructures, vol. 11, no. 1, pp. 25-34, 2003.

[68] M. E. El-Khouly, O. Ito, P. M. Smith, and F. D'Souza, "Intermolecular and supramolecular photoinduced electron transfer processes of fullerene-porphyrin/phthalocyanine systems," Journal of Photochemistry and Photobiology C: Photochemistry Reviews, vol. 5, no. 1, pp. 79-104, 2004.

[69] M. Alvaro, P. Atienzar, P. de La Cruz et al., "Synthesis, photochemistry, and electrochemistry of single-wall carbon nanotubes with pendent pyridyl groups and of their metal complexes with zinc porphyrin. Comparison with pyridyl-bearing fullerenes," Journal of the American Chemical Society, vol. 128, no. 20, pp. 6626-6635, 2006.

[70] S. Tagliapietra, G. Cravotto, E. Gaudino, S. Visentin, and V. Mussi, "Functionalization of single-walled carbon nanotubes through 1,3-cycloaddition of carbonyl ylides under microwave irradiation," Synlett, vol. 23, no. 10, Article ID ST-2012-B0134-L, pp. 1459-1462, 2012.

[71] K. Martina, F. Baricco, G. Berlier, M. Caporaso, and G. Cravotto, "Efficient green protocols for the preparation of highly functionalized $\beta$-cyclodextrin grafted silica," ACS-Sustainable Chemistry and Engineering, vol. 2, no. 11, pp. 2595-2603, 2014. 

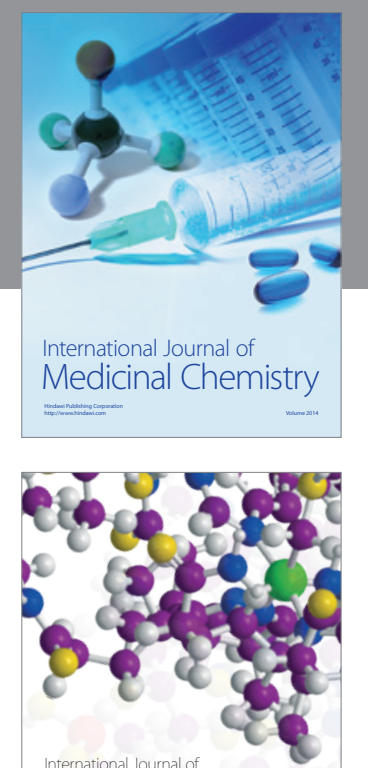

\section{Carbohydrate} Chemistry

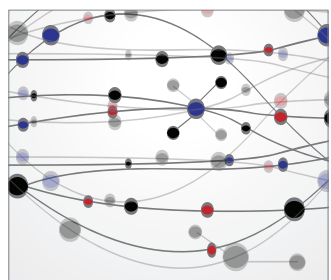

The Scientific World Journal
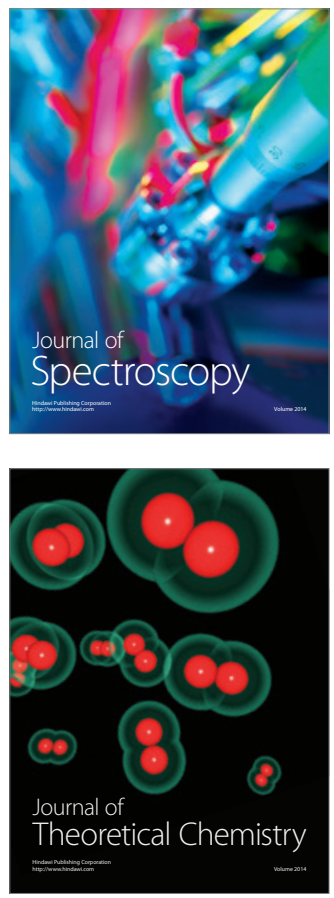
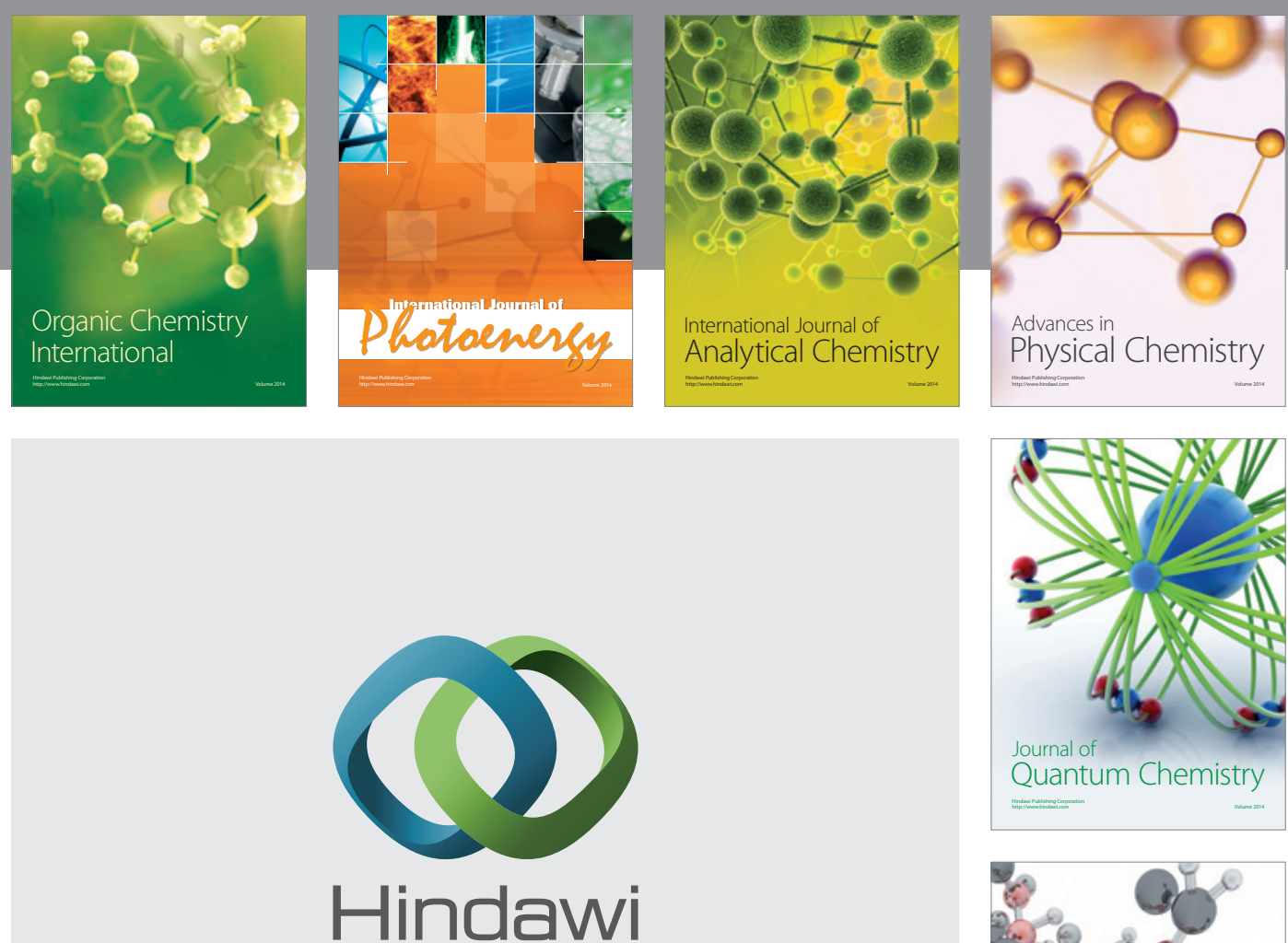

Submit your manuscripts at

http://www.hindawi.com

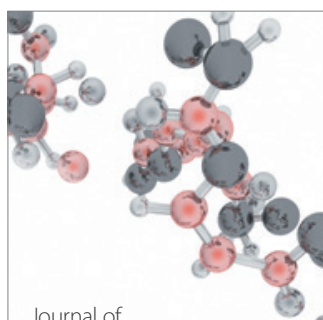

Analytical Methods

in Chemistry

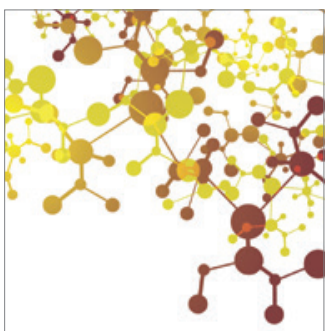

Journal of

Applied Chemistry

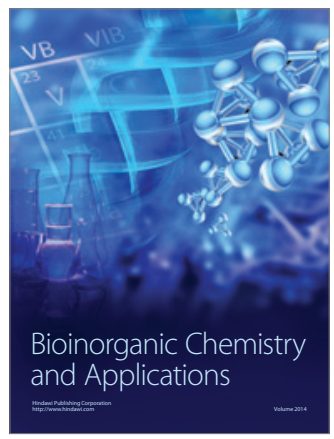

Inorganic Chemistry
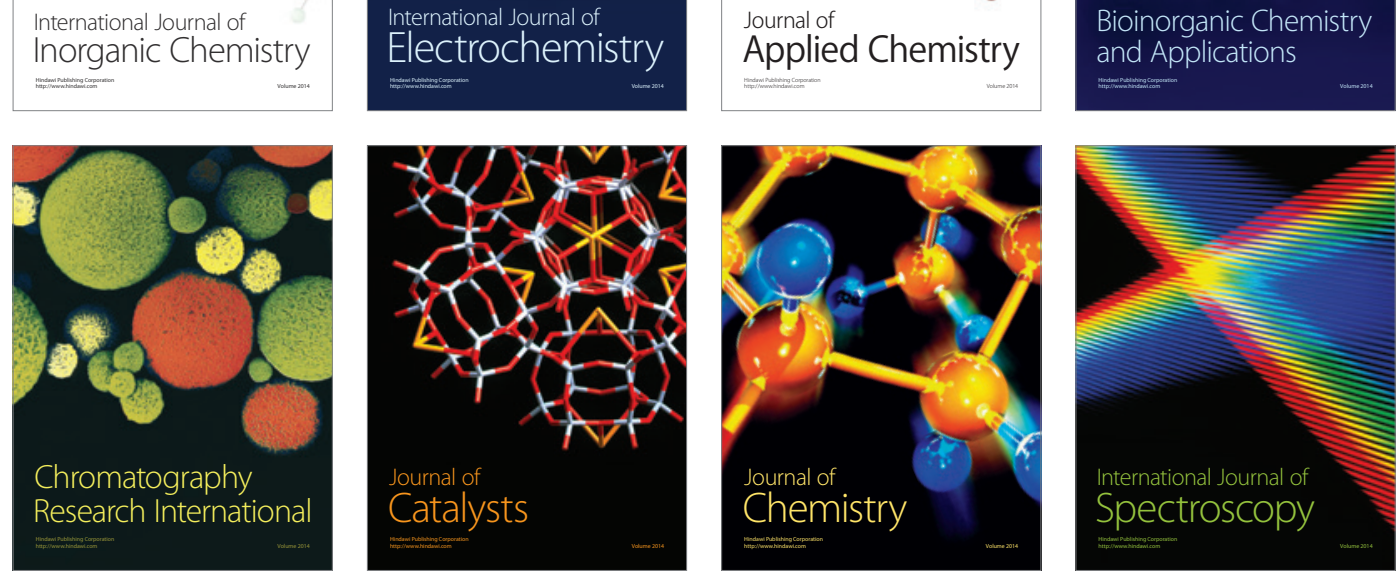